\title{
Leader-Following Consensus Stability of Discrete-Time Linear Multiagent Systems with Observer-Based Protocols
}

\author{
Bingbing Xu, ${ }^{1}$ Lixin Gao, ${ }^{1}$ Yan $\mathrm{Zhang}^{1}$ and Xiaole $\mathrm{Xu}{ }^{2}$ \\ ${ }^{1}$ Institute of Intelligent Systems and Decision, Wenzhou University, Zhejiang 325035, China \\ ${ }^{2}$ Wenzhou Vocational College of Science \& Technology, Zhejiang 325006, China \\ Correspondence should be addressed to Lixin Gao; gao-lixin@163.com
}

Received 16 February 2013; Accepted 26 August 2013

Academic Editor: Pagavathi Balasubramaniam

Copyright (c) 2013 Bingbing Xu et al. This is an open access article distributed under the Creative Commons Attribution License, which permits unrestricted use, distribution, and reproduction in any medium, provided the original work is properly cited.

\begin{abstract}
We consider the leader-following consensus problem of discrete-time multiagent systems on a directed communication topology. Two types of distributed observer-based consensus protocols are considered to solve such a problem. The observers involved in the proposed protocols include full-order observer and reduced-order observer, which are used to reconstruct the state variables. Two algorithms are provided to construct the consensus protocols, which are based on the modified discrete-time algebraic Riccati equation and Sylvester equation. In light of graph and matrix theory, some consensus conditions are established. Finally, a numerical example is provided to illustrate the obtained result.
\end{abstract}

\section{Introduction}

In recent decades, the cooperate and control problem of distributed dynamic systems has been a challenging research field, owing to its widespread applications in many areas such as swarm of animals [1], collective motion of particles [2], schooling for underwater vehicles $[3,4]$, neural networks $[5,6]$, and distributed sensor networks [7].

The consensus problem, as one fundamental problem for coordinated control of multiagent systems, has gained significant attention from different research communities. Consensus problem considers how to design an information interaction protocol between agents and requires all agents to converge to a common value $[8,9]$. Based on matrix theory, algebraic graph theory, and control theory, many researchers have acquired abundant results in studying consensus problem of multiagent systems. In [10], the authors proposed a general framework for consensus problem in fixed and switching networks and gave solution to the case with communication time delays. Olfati-Saber et al. established a general model for consensus problems of the multiagent systems and introduced Lyapunov method to reveal the contract with the connectivity of the graph theory and the stability of the system in [11]. Sometimes, it is better to consider a tracking consensus problem by adding a leader which can make all agents reach a command trajectory with any initial condition [12]. The leader-following consensus problem has been addressed in many references [13-17].

Many proposed distributed consensus protocols need to know neighbors' state information, but it may be difficult to measure this information. To make the system achieve consensus, it often contains an observer in the control protocol, which is used to estimate those unmeasurable state variables. The distributed observer-based control laws were proposed to solve first-order and second-order multiagent consensus problems in $[12,17]$. To estimate the general active leader's unmeasurable state variables, [18] proposed a distributed algorithm for first-order agent, and [19] extended the results of [18] to the time-delay case. The distributed observer-based consensus protocols were addressed to solve multiagent consensus with general linear or linearized agent dynamics in [17, 20-24]. In [25], the author proposed an observer-type consensus protocol to the consensus problem for a class of fractional-order uncertain multiagent systems with general linear dynamics. In [26], the authors proposed distributed reduced-order observer-based protocols to solve consensus problem, which were generalized to solve leader-following consensus problem under switching topology by [27]. 
The observer-based consensus protocol can be viewed as a special case of the dynamic compensation method, which has been investigated by [28-30].

Discrete-time dynamic systems are commonly involved in the neural network, sampled control, signal filters, and state estimators. The discrete-time neural network was studied by [31-33]. The sampled-data discrete-time coordination of multiagent systems was investigated in $[16,34,35]$. The first-order discrete-time consensus has been investigated by $[8,9,36-38]$. In [39], the authors discussed discretetime second-order consensus protocols for dynamics with nonuniform and time-varying communication delays under dynamically switching topology. The distributed $H_{\infty}$ consensus problem was studied in [30] to solve multiagent consensus problem with discrete-time high-dimensional linear coupling dynamics subjected to external disturbances. The distributed state-feedback protocols for linear discrete-time multiagent were proposed in $[40,41]$. The distributed observer-based protocol was proposed to solve leader-following consensus problem with linear discrete-time dynamics in [23, 42, 43].

Motivated by the above works, we focus our research on a group of agents with discrete-time high-dimensional linear coupling dynamics and directed interaction topology. We propose distributed observer-based protocols for leaderfollowing multiagent systems. The full-order observer and reduced-order observer are adopted to reconstruct the state variables. Contrary to [23] and [40], the gain matrix design approach used in this paper is based on the modified discretetime algebraic Riccati equations (MDARE) but not the normal discrete-time algebraic Riccati equations. The proposed design method must be feasible if spectral radius of system matrix is not greater than 1 . Of course, the proposed design method can be used to construct the consensus protocols provided by [23] and [40]. Further, the separation principle is shown to be valid, from which we can establish consensus condition for closed-loop multiagent systems.

This paper is organized as follows. Section 2 presents the related notations and the problem formulated with graph theory. In Section 3, the distributed state feedback design is considered. In Sections 4 and 5, the distributed full-order and reduced-order observer-based consensus protocols are proposed, respectively, which are the main results of this paper. Section 6 presents a simulation example to illustrate our established results. Finally, the conclusion is given in Section 7.

\section{Preliminaries and Problem Formulation}

2.1. Notations and Graph Theory. $\operatorname{Re}(\xi)$ denotes the real part of $\xi \in C$. Let $R^{m \times n}$ and $C^{m \times n}$ be the set of $m \times n$ real matrices and complex matrices, respectively. $\mathbf{1}_{n} \in R^{n}$ is the column vector with all components equal to one. Let $I$ be the identity matrix with compatible dimension. For a given matrix $A, a_{i j}$ represents its element of $i$ th row and jth column, $A^{T}$ denotes its transpose, and $A^{H}$ denotes its conjugate transpose. A matrix is said to be Schur-stable if all its eigenvalues are inside unit circle. $\rho(A)$ represents the spectral radius of matrix $A$. $\lambda_{\max }(A)$ and $\lambda_{\min }(A)$ represent its maximum and minimum eigenvalues of symmetric matrix $A$, respectively. For symmetric matrices $A$ and $B, A>B$ means that $A-B$ is positive definite, that is, $A-B>0$. $\otimes$ denotes Kronecker product, which satisfies $(A \otimes B)(C \otimes D)=$ $(A C) \otimes(B D)$.

We describe the interaction relationship among $n$ agents by a simple weighted diagraph $\mathscr{G}=\{\mathscr{V}, \mathcal{\varepsilon}, W\}$, where $\mathscr{V}=$ $\left\{v_{1}, v_{2}, \ldots, v_{n}\right\}$ is the set of vertices and $\varepsilon \subset \mathscr{V} \times \mathscr{V}$ is the set of edges. If $\left(v_{i}, v_{j}\right) \in \varepsilon$, the vertex $v_{j}$ is called a neighbor of vertex $v_{i}$, and the index set of neighbors of vertex $v_{i}$ is denoted by $\mathcal{N}_{i}=\left\{j \mid\left(v_{i}, v_{j}\right) \in \varepsilon\right\} . W=\left[w_{i j}\right]_{n \times n}$ represents weighted adjacency matrix associated with graph $\mathscr{G}$, where $w_{i j}>0$ if $\left(v_{i}, v_{j}\right) \in \varepsilon$ and $w_{i j}=0$ otherwise. The degree matrix $D=\operatorname{diag}\left\{d_{1}, d_{2}, \ldots, d_{n}\right\}$ of digraph $\mathscr{G}$ is a diagonal matrix with diagonal elements $d_{i}=\sum_{j=1}^{n} w_{i j}$. Then, the Laplacian matrix of $\mathscr{G}$ is defined as $L=D-W . v_{i}$ is called globally reachable node if there exists at least a directed path from every other node to node $v_{i}$ in digraph $\mathscr{G}$. A directed graph $\mathscr{G}$ has a globally reachable node if and only if there exists a directed spanning tree in $\mathscr{G}$ (see [9]).

For a multiagent system with leader (labeled as 0 ), the interaction topology is expressed by graph $\widehat{\mathscr{G}}$, which contains graph $\mathscr{G}$ and vertex $v_{0}$ and edges from other vertices to vertex $v_{0}$. Let $g_{i}, i=1,2, \ldots, n$, be weight of $\left(v_{i}, v_{0}\right), g_{i}>0$ if $\left(v_{i}, v_{0}\right)$ is an edge of graph $\widehat{G}$ and $g_{i}=0$ otherwise. Let $G_{d}=\operatorname{diag}\left\{g_{1}, g_{2}, \ldots, g_{n}\right\}$. The matrix $L+G_{d}$ has the following property.

Lemma 1 (see [13]). Matrix $L+G_{d}$ is positive stable if and only if graph $\widehat{\mathscr{G}}$ has a directed spanning tree with root $v_{0}$.

2.2. Problem Formulation. Consider the multiagent system which is composed of $n$ identical following agents and a leader. Each following agent has dynamics modeled by the discrete-time linear system:

$$
\begin{gathered}
x_{i}(k+1)=A x_{i}(k)+B u_{i}(k), \\
y_{i}(k)=C x_{i}(k),
\end{gathered}
$$

where $x_{i}(k) \in R^{m}, u_{i}(k) \in R^{p}$, and $y_{i}(k) \in R^{q}$ are, respectively, the state variable, control input, and measured output of agent $i$.

The dynamics of the leader is given as

$$
\begin{gathered}
x_{0}(k+1)=A x_{0}(k), \\
y_{0}(k)=C x_{0}(k),
\end{gathered}
$$

where $x_{0}(k)$ is the state and $y_{0}(k)$ is the measured output of the leader. The leaderless consensus problem for multiagent system has been investigated by $[26,28,44]$, which require the system matrix $A$ to be Schur-stable. There is not such requirement to $A$ in this paper. $A, B, C$ are constant matrices with compatible dimensions. It is assumed that $(A, B$, and $C)$ is stabilizable and detectable.

The $x_{0}(k)$ is often called as "consensus reference state" and assumed to be available only to a subgroup of the followers. The main objective of leader-following consensus problem is 
to design distributed consensus protocol to make multiagent system achieve consensus.

Definition 2. The leader-following multiagent system is said to achieve consensus if the state variables of all following agents satisfy $\lim _{k \rightarrow \infty}\left(x_{i}(k)-x_{0}(k)\right)=0, i=1,2, \ldots, n$ for any initial state. One says that the protocol $u_{i}(k)$ can solve the leader-following consensus problem if the closed-loop system achieves consensus.

2.3. Preliminary Results. In this subsection, we introduce some preliminary results which will be used to establish our main results. Consider the following MDARE:

$$
A^{T} P A-P-\delta A^{T} P B\left(I+B^{T} P B\right)^{-1} B^{T} P A+Q=0,
$$

where $Q$ is any given positive definite matrix. Since $Q$ is positive definite, $\left(A, Q^{1 / 2}\right)$ must be detectable. The solvability of the MDARE is addressed by the following lemma.

Lemma 3 (see $[45,46]$ ). If $\left(A, Q^{1 / 2}\right)$ is detectable, $(A, B)$ is stabilizable, then there exists a $\delta_{c} \in[0,1)$ such that the modified discrete time algebraic Riccati equation (3) has a unique positive-definite solution $P$ for any $\delta_{c}<\delta \leq 1$. Furthermore, $P=\lim _{k \rightarrow \infty} P_{k}$ for any initial condition $P_{0} \geq 0$, where $P_{k}$ satisfies

$$
P_{k+1}=A^{T} P_{k} A-\delta A^{T} P_{k} B\left(I+B^{T} P_{k} B\right)^{-1} B^{T} P_{k} A+Q .
$$

Remark 4. The MDARE (3) is reduced, respectively, to the well-known discrete-time Riccati equation (DARE) and Stain equation as $\delta=1$ and $\delta=0$. The Stain equation has a unique positive-definite solution if $A$ is Schur-stable. It is well known that DARE has a unique positive-definite solution if $(A, B)$ is stablizable. If the involved matrix $A$ is not Schur-stable, it is easy to see that $0<\delta_{c} \leq 1$. More details for issue $\delta_{c}$ can be referenced to [45]. Moreover, if the matrix $A$ has no eigenvalues with magnitude larger than 1 and $(A, C)$ is detectable, MDARE (3) has a unique positivedefinite solution $P$ for any $\delta$ satisfying $0<\delta \leq 1$.

Lemma 5. For a given $\delta$ satisfying $\delta_{c}<\delta \leq 1$, let $P$ be the unique positive-definite solution of the MDARE (3). Choose a feedback matrix $K=\left(I+B^{T} P B\right)^{-1} B^{T} P A$. Then, $A-s B K$ is Schur-stable for any $s \in \bar{C}(1, \sqrt{1-\delta})$.

Proof. From the MDARE (3), we have

$$
\begin{aligned}
&(A-s B K)^{*} P(A-s B K)-P \\
&=A^{T} P A-\left(s+s^{*}\right) A^{T} P B\left(I+B^{T} P B\right)^{-1} B^{T} P A \\
&+s s^{*} K^{T} B^{T} P B K-P \\
&= A^{T} P A-P-\left(s+s^{*}-s s^{*}\right) A^{T} P B\left(I+B^{T} P B\right)^{-1} \\
& \times B^{T} P A-|s|^{2} K^{T} K
\end{aligned}
$$

$$
\begin{aligned}
= & A^{T} P A-P-\left(1-|s-1|^{2}\right) A^{T} P B\left(I+B^{T} P B\right)^{-1} \\
& \times B^{T} P A-|s|^{2} K^{T} K \\
\leq & A^{T} P A-P-\delta A^{T} P B\left(I+B^{T} P B\right)^{-1} B^{T} P A \\
\leq & -Q<0 .
\end{aligned}
$$

Thus, we know that if $|s-1| \leq \sqrt{1-\delta}, A-s B K$ is Schurstable.

\section{Distributed State Feedback Design}

In this section, we investigate the multiagent consensus via state variable feedback control, which has been addressed by [23]. Here, we also use the control protocol proposed by [23] and provide a new design approach to construct the feedback gain matrix.

The neighborhood disagreement error of agent $i$ is defined as

$$
\xi_{i}(k)=\sum_{j \in \mathcal{N}_{i}} w_{i j}\left(x_{j}(k)-x_{i}(k)\right)+g_{i}\left(x_{0}(k)-x_{i}(k)\right) .
$$

Consider the following distributed state feedback protocol for agent $i$ :

$$
u_{i}(k)=c_{1}\left(1+d_{i}+g_{i}\right)^{-1} K \xi_{i}(k),
$$

where $d_{i}=\sum_{j \in \mathcal{N}_{i}} w_{i j}, c_{1}$ is the coupling strength and $K$ is a feedback gain matrix, which will be determined later.

Denote $e_{i}(k)=x_{i}(k)-x_{0}(k)$ and $e(k)=\left[e_{1}^{T}(k), e_{2}^{T}(k)\right.$, $\left.\ldots, e_{n}^{T}(k)\right]^{T}$. Then, we can derive that the close loop system has the global tracking error dynamics as follows [23]

$$
e(k+1)=\left[I_{n} \otimes A-c_{1} \Gamma \otimes(B K)\right] e(k),
$$

where $\Gamma=\left(I+D+G_{d}\right)^{-1}\left(L+G_{d}\right)$.

Definition 6 (see [23]). A covering circle $\bar{C}\left(c_{0}, r_{0}\right)$ related to matrix $\Gamma$ is a closed circle in the complex plane centered at $c_{0} \in R$ and $\lambda_{i} \in \bar{C}\left(c_{0}, r_{0}\right)$ for all $i=1,2, \ldots, n$.

Then, we provide a new design technique to construct feedback gain matrix $K$, which is presented in the following theorem.

Theorem 7. For multiagent system (1) and (2), assume that the interconnection topology $\widehat{\mathscr{G}}$ has a directed spanning tree with root $v_{0}$. If there exists a covering circle $\bar{C}\left(c_{0}, r_{0}\right)$ such that

$$
0<\frac{r_{0}}{c_{0}}<\sqrt{1-\delta_{c}}
$$

then there must exist fitted $c_{1}$ and $K$ such that the global tracking error dynamics (8) is asymptotically stable. Furthermore, by taking $\delta$ which satisfies

$$
\frac{r_{0}}{c_{0}} \leq \sqrt{1-\delta}<\sqrt{1-\delta_{c}}
$$


and solving the MDARE (3) to get the unique positive-definite solution $P$, the feedback matrix $K$ and the coupling strength $c_{1}$ can be chosen as

$$
\begin{gathered}
K=\left(I+B^{T} P B\right)^{-1} B^{T} P A, \\
c_{1}=\frac{1}{c_{0}} .
\end{gathered}
$$

Proof. From (10), we know $\delta>\delta_{c}$, which means that the MDARE (3) has a unique positive-definite solution $P$. Any $\lambda_{i}$ satisfies $\left|\lambda_{i}-c_{0}\right| \leq r_{0}$. Thus, $\left|c_{1} \lambda_{i}-1\right| \leq r_{0} / c_{0}<\sqrt{1-\delta}$. According to Lemma 5, all $A-c_{1} \lambda_{i} B K, i=1,2, \ldots, n$ are Schur-stable.

Let $U$ be a Schur transformation matrix of $\Gamma$ such that

$$
U^{T} \Gamma U=\left[\begin{array}{cccc}
\lambda_{1} & * & \cdots & * \\
0 & \lambda_{2} & \cdots & * \\
\vdots & \vdots & \ddots & \vdots \\
0 & 0 & \cdots & \lambda_{n}
\end{array}\right] .
$$

Then, we have

$$
\begin{aligned}
& (U \times I)^{T}\left[I_{n} \otimes A-c_{1} \Gamma \otimes(B K)\right](U \times I) \\
& =\left[\begin{array}{cccc}
A-c_{1} \lambda_{1} B K & * & \cdots & * \\
0 & A-c_{1} \lambda_{2} B K_{c} & \cdots & * \\
\vdots & \vdots & \ddots & \vdots \\
0 & 0 & \cdots & A-c_{1} \lambda_{n} B K_{c}
\end{array}\right] .
\end{aligned}
$$

Certainly, $U \otimes I$ is also a unitary matrix. Matrix $\left[I_{n} \otimes A-\right.$ $\left.c_{1} \Gamma \otimes(B K)\right]$ is Schur-stable if and only if all $A-c_{1} \lambda_{i} B K, i=$ $1,2, \ldots, n$ are Schur-stable. Now, the proof is completed.

Remark 8. From condition (9), it is required that $0<c_{0}<r_{0}$, which means that the covering circle should be located in the open right half plane. Moreover, the small enough $r_{0} / c_{0}$ will guarantee that the MDARE (3) is solvable, which is the key point in the proposed design approach. The weight parameter in the feedback law (7) need not take $c_{1}\left(1+d_{i}+g_{i}\right)^{-1}$, which can be selected as $c_{1}\left(d_{i}+g_{i}\right)^{-1}, c_{1}$, and so on as long as there exists a covering circle for the related matrix $c_{1} \Gamma$ that satisfies the condition (9).

Next, we will discuss the covering circle of the matrix $c_{1} \Gamma$. Based on Gershgorin disk theorem [47], all the eigenvalues of $\left(I+D+G_{d}\right)^{-1}(I+W)$ are located in the union of $n$ discs:

$$
\bigcup_{j=1}^{n}\left\{s \in C:\left|s-\frac{1}{1+d_{i}+g_{i}}\right| \leq \frac{d_{i}}{1+d_{i}+g_{i}}\right\} \text {. }
$$

It is easy to see that this union is included in a unit circle $\{s:|s| \leq 1\}$ and the circular boundaries of the union of $n$ discs have only one intersection with the circle at $s=1$. If the interconnection topology $\widehat{\mathscr{G}}$ has a directed spanning tree with root $v_{0}$, we know that $L+G_{d}$ is nonsingular, and then, $\Gamma$ is nonsingular too. Noting that $\left(I+D+G_{d}\right)^{-1}(I+W)=I-\Gamma$, then we know that all eigenvalues of matrix $\left(I+D+G_{d}\right)^{-1}(I+W)$ are not equal to 1 . Thus, all eigenvalues of matrix $\Gamma$ can be covered by circle $\bar{C}\left(1, r_{0}\right)$ with $r_{0}<1$. On the other hand, it is necessary to assume that the interconnection topology $\widehat{\mathscr{G}}$ has a directed spanning tree with root $v_{0}$. Otherwise, there exists at least one agent which cannot get the leader's information directly and indirectly. Certainly, if $A$ is not Schur-stable, those agents cannot track the leader with some initial values. From this point, the assumption that the interconnection topology $\widehat{\mathscr{G}}$ has a directed spanning tree with root $v_{0}$ is necessary.

An interesting special case is that matrix $A$ has no eigenvalues with magnitude larger than 1 , that is, $\rho(A) \leq$ 1. The well-known second-order discrete-time multiagent system

$$
\begin{aligned}
& x_{i}(k+1)=x_{i}(k)+v_{i}(k), \\
& v_{i}(k+1)=v_{i}(k)+u_{i}(k),
\end{aligned}
$$

has been addressed in many references $[34,38]$. The system matrix $A$ of second-order discrete-time multiagent system is $\left[\begin{array}{ll}1 & 1 \\ 0 & 1\end{array}\right]$, which has no eigenvalues with magnitude larger than 1 .

According to Theorem 7, we present the following corollary for this special case.

Corollary 9. For multiagent system (1) and (2) with $\rho(A) \leq$ 1 , assume that the interconnection topology $\widehat{\mathscr{G}}$ has a directed spanning tree with root $v_{0}$. Take $\delta=1-\max _{i}\left\{\left|\lambda_{i}-1\right|^{2}\right\}$, and solve the MDARE (3) to get the unique positive-definite solution $P$. Choose $K=\left(I+B^{T} P B\right)^{-1} B^{T} P A$ and $C_{1}=1$. Then, the distributed feedback control (7) guarantees that all following agents can track leader.

Proof. According to Remark 4, we know $\delta_{c}=0$ if $\rho(A) \leq 1$. Select $\delta=1-\max _{i}\left\{\left|\lambda_{i}-1\right|^{2}\right\}$. From above analysis, we know that $\delta>0$ and $C(1, \delta)$ are a covering circle. Thus, the MDARE (3) is solvable. According to Theorem 7, we can obtain the corollary directly.

\section{Consensus Protocol Design with Full-Order Observer}

In many applications, each agent only accesses the neighbor's output variable. To solve leader-following consensus problem, we propose a new observer-based consensus protocol for agent $i$, which consists of a distributed estimation law and a feedback control law.

(i) Local estimation law for agent $i$ :

$$
\begin{gathered}
z_{i}(k+1)=F z_{i}(k)+G y_{i}(k)+T B u_{i}(k), \\
\widehat{x}_{i}(k)=T^{-1} z_{i}(k),
\end{gathered}
$$

where $z_{i}(k) \in R^{m}$ is the protocol state, $\widehat{x}(k)$ is the constructed variable to estimate $x_{i}(k)$, and $F \in R^{m \times m}$, $G \in R^{m \times q}$, and $T \in R^{m \times m}$ are the designed parameter matrices. 
(ii) Neighbor-based feedback control law for agent $i$ :

$$
u_{i}(k)=c_{1}\left(1+d_{i}+g_{i}\right)^{-1} K \eta_{i}(k)
$$

where the neighborhood disagreement observer error $\eta_{i}(k)$ of agent $i$ is denoted as

$$
\eta_{i}(k)=\sum_{j \in \mathcal{N}_{i}} w_{i j}\left(\widehat{x}_{j}(k)-\widehat{x}_{i}(k)\right)+g_{i}\left(x_{0}(k)-\widehat{x}_{i}(k)\right)
$$

and $K$ is a given feedback gain matrix.

Next, an algorithm is provided to select the parameter matrices used in estimation law (16).

Algorithm 10. Given that $(A, C)$ is observable. The parameter matrices $F, G$, and $T$ used in estimation law (16) can be constructed as follows.

(1) Select a Schur-stable $m \times m$ matrix $F$ with a set of desired eigenvalues that contain no eigenvalues in common with those of $A$.

(2) Select $G \in R^{m \times q}$ randomly such that $(F, G)$ is controllable.

(3) Solve Sylvester equation

$$
T A-F T=G C
$$

to get a nonsingular solution $T$. If $T$ is singular, select another $G$ until $T$ is nonsingular.

Denote $\widehat{e}_{i}(k)=z_{i}(k)-T x_{i}(k)$ and $\hat{e}(k)=\left[\hat{e}_{1}^{T}(k), \hat{e}_{2}^{T}(k)\right.$, $\left.\ldots, \hat{e}_{n}^{T}(k)\right]^{T}$. Then, after manipulations and combining (1) and (16), we can obtain

$$
\begin{aligned}
\widehat{e}_{i}(k+1) \\
=z_{i}(k+1)-T x_{i}(k+1) \\
=F z(k)+G y_{i}(k)+T B u_{i}(k)-T A x_{i}(k)-T B U_{i}(k) \\
=F \widehat{e}_{i}(k)+(F T+G C-T A) x_{i}(k) \\
=F \widehat{e}_{i}(k) .
\end{aligned}
$$

For tracking error $e_{i}(k)=x_{i}(k)-x_{0}(k)$, we have

$$
\begin{aligned}
e_{i}(k+1) & \\
= & A e_{i}(k+1)+c_{1}\left(1+d_{i}+g_{i}\right)^{-1} K \eta_{i}(k) \\
= & A e_{i}(k+1)+c_{1}\left(1+d_{i}+g_{i}\right)^{-1} K \xi_{i}(k) \\
& +c_{1}\left(1+d_{i}+g_{i}\right)^{-1} K\left[\eta_{i}(k)-\xi_{i}(k)\right] \\
= & A e_{i}(k+1)-c_{1}\left(1+d_{i}+g_{i}\right)^{-1}
\end{aligned}
$$

$$
\begin{aligned}
& \times\left[K \sum_{j \in \mathcal{N}_{i}} w_{i j}\left(e_{i}(k)-e_{j}(k)\right)+g_{i} e_{i}\right] \\
& +c_{1}\left(1+d_{i}+g_{i}\right)^{-1} K T^{-1} \\
& \times\left[\sum_{j \in \mathcal{N}_{i}} w_{i j}\left(\widehat{e}_{i}(k)-\widehat{e}_{j}(k)\right)+g_{i} \widehat{e}_{i}\right] .
\end{aligned}
$$

From (20) and (21), the error dynamics of closed-loop system will be expressed as

$$
\begin{aligned}
& {\left[\begin{array}{c}
e(k+1) \\
\widehat{e}(k+1)
\end{array}\right]} \\
& =\left[\begin{array}{cc}
I_{n} \otimes A-c_{1} \Gamma \otimes(B K) & c_{1} \Gamma \otimes\left(B K T^{-1}\right) \\
0 & I \otimes F
\end{array}\right] \\
& \quad \times\left[\begin{array}{l}
e(k) \\
\hat{e}(k)
\end{array}\right] .
\end{aligned}
$$

Obviously, the error dynamics system (22) is Schur-stable if and only if $I_{n} \otimes A-c_{1} \Gamma \otimes(B K)$ and $I \otimes F$ are Schur-stable. Similar to Theorem 7, we present the following theorem directly, and the proof is omitted.

Theorem 11. For multiagent system (1) and (2), assume that the interconnection topology $\widehat{\mathscr{G}}$ has a directed spanning tree with root $v_{0}$. If there exists a covering circle $\bar{C}\left(c_{0}, r_{0}\right)$ such that

$$
0<\frac{r_{0}}{c_{0}}<\sqrt{1-\delta_{c}}
$$

then the distributed observer-based protocols (16) and (17) can solve the discrete-time leader-following consensus problem. Furthermore, the parameter matrices $F, G$, and $T$ used in observer (16) are constructed by Algorithm 10. By taking $\delta$ satisfied

$$
\frac{r_{0}}{c_{0}} \leq \sqrt{1-\delta}<\sqrt{1-\delta_{c}}
$$

and solving the MDARE (3) to get the unique positive-definite solution $P$, the feedback matrix $K$ and the coupling strength $c_{1}$ can be chosen as

$$
\begin{gathered}
K=\left(I+B^{T} P B\right)^{-1} B^{T} P A, \\
c_{1}=\frac{1}{c_{0}} .
\end{gathered}
$$

Remark 12. Of course, when system matrix $A$ satisfies $\rho(A) \leq$ 1 , we can also establish similar corollaries as Corollary 9 in this section and the next section. In [23], three different observer/controller architectures are proposed for dynamic output feedback regulator design. Besides design feedback matrix $K$, another key technique is to choose an observer gain matrix $L$ which makes $I_{n} \otimes A-c_{1} \Gamma \otimes(L C)$ Schur-stable. By using duality property, solve the following MDARE:

$$
A P A^{T}-P-\delta A P C^{T}\left(I+C P C^{T}\right)^{-1} C P A^{T}+Q=0
$$


to get the unique positive definite solution $P$. Then, the observer gain matrix $L$ is chosen as $L=A P C^{T}\left(I+C P C^{T}\right)^{-1}$. Thus, the proposed design method in this paper can also be applied to construct the protocols proposed by [23]. In this paper, we propose two new observer/controller architectures, which will replenish cooperative observer and regulator theory. Contrary to [23], our proposed approach must be feasible if system matrix $A$ satisfies $\rho(A) \leq 1$.

\section{Consensus Protocol Design with Reduced-Order Observer}

In this section, we assume that $C$ has full row rank, that is, $\operatorname{Rank}(C)=q$. The following reduced-order observerbased consensus protocol, which consists of a reduced-order estimation law and a feedback control law, is proposed for agent $i$.

(i) Local reduced-order estimation law for agent $i$ :

$$
v_{i}(k+1)=F v_{i}(k)+G y_{i}(k)+T B u_{i}(k),
$$

where $v_{i}(k) \in R^{m-q}$ is the protocol state, $F \in$ $R^{m-q \times m-q}$, and $G \in R^{m-q \times q}$ and $T \in R^{m-q \times m}$ are parameter matrices.

(ii) Neighbor-based feedback control law for agent $i$ :

$$
u_{i}(k)=c_{1}\left(1+d_{i}+g_{i}\right)^{-1} K \zeta_{i}(k),
$$

where the disagreement error $\zeta_{i}(k)$ of agent $i$ is given as

$$
\begin{aligned}
\zeta_{i}(k)= & Q_{1}\left[\sum_{j \in \mathcal{N}_{i}} w_{i j}\left(y_{j}(k)-y_{i}(k)\right)+g_{i}\left(y_{0}(k)-y_{i}(k)\right)\right] \\
& +Q_{2}\left[\sum_{j \in \mathcal{N}_{i}} w_{i j}\left(v_{j}(k)-v_{i}(k)\right)+g_{i}\left(T x_{0}(k)-v_{i}(k)\right)\right],
\end{aligned}
$$

and $K$ is a gain matrix.

Similarly, an algorithm is presented to design the same parameter matrices used in the protocols (27) and (28).

Algorithm 13. Given that $(A, C)$ is observable. The parameter matrices $F, G, T, Q_{1}$, and $Q_{2}$ can be constructed as follows.

(1) Select a Schur matrix $F \in R^{(m-q) \times(m-q)}$ with a set of desired eigenvalues that contain no eigenvalues in common with those of $A$.

(2) Select $G \in R^{(m-q) \times q}$ randomly such that $(F, G)$ is controllable.

(3) Solve Sylvester equation

$$
T A-F T=G C
$$

to get the unique solution $T$, which satisfies that $\left[\begin{array}{c}C \\ T\end{array}\right]$ is nonsingular. If $\left[\begin{array}{c}C \\ T\end{array}\right]$ is singular, go back to step (2) to select another $G$ until $\left[\begin{array}{c}C \\ T\end{array}\right]$ is nonsingular.
(4) Compute matrices $Q_{1} \in R^{m \times q}$ and $Q_{2} \in R^{m \times(m-q)}$ by $\left[\begin{array}{ll}Q_{1} & Q_{2}\end{array}\right]=\left[\begin{array}{c}C \\ T\end{array}\right]^{-1}$.

Now, we present the result related to reduced-order observer.

Theorem 14. For multiagent system (1) and (2), assume that the interconnection topology $\widehat{\mathscr{G}}$ has a directed spanning tree with root $v_{0}$. If there exists a covering circle $\bar{C}\left(c_{0}, r_{0}\right)$ such that

$$
0<\frac{r_{0}}{c_{0}}<\sqrt{1-\delta_{c}}
$$

then the distributed observer-based protocols (16) and (17) can solve the discrete-time leader-following consensus problem. Furthermore, the parameter matrices $F, G, T, Q_{1}$, and $Q_{2}$ used in protocols (27) and (28) are constructed by Algorithm 13. By taking $\delta$ which satisfies

$$
\frac{r_{0}}{c_{0}} \leq \sqrt{1-\delta}<\sqrt{1-\delta_{c}}
$$

and solving the MDARE (3) to get the unique positive-definite solution $P$, the feedback matrix $K$ and the coupling strength $c_{1}$ can be chosen as

$$
\begin{gathered}
K=\left(I+B^{T} P B\right)^{-1} B^{T} P A, \\
c_{1}=\frac{1}{c_{0}} .
\end{gathered}
$$

Proof. To analyze convergence, denote $e_{i}(k)=x_{i}(k)-x_{0}(k)$ and $\varepsilon_{i}=v_{i}(k)-T x_{0}(k)$. Then, the dynamics of $e_{i}(k)$ and $\varepsilon_{i}(k)$ satisfy

$$
\begin{aligned}
e_{i}(k+1)= & A e_{i}(k)-c_{1}\left(1+d_{i}+g_{i}\right)^{-1} B K Q_{1} C \\
& \times\left[\sum_{j \in \mathcal{N}_{i}} w_{i j}\left(e_{i}(k)-e_{j}(k)\right)+g_{i} e_{i}(k)\right] \\
& -c_{1}\left(1+d_{i}+g_{i}\right)^{-1} K Q_{2} \\
& \times\left[\sum_{j \in \mathcal{N}_{i}} w_{i j}\left(\varepsilon_{i}(k)-\varepsilon_{j}(k)\right)+g_{i} \varepsilon_{i}(k)\right], \\
\varepsilon_{i}(k+1)= & v_{i}(k+1)-T x_{0}(k+1) \\
= & F v_{i}(k)+G C x_{i}(k)-T A x_{0}(k) \\
& -c_{1}\left(1+d_{i}+g_{i}\right)^{-1} T B K Q_{1} C \\
& \times\left[\sum_{j \in \mathscr{N}_{i}(t)} w_{i j}\left(x_{i}-x_{j}\right)+g_{i}\left(x_{i}-x_{0}\right)\right] \\
& -c_{1}\left(1+d_{i}+g_{i}\right)^{-1} T B K Q_{2} \\
& \times\left[\sum_{j \in \mathcal{N}_{i}} w_{i j}\left(v_{i}-v_{j}\right)+g_{i}\left(v_{i}-T x_{0}\right)\right] \\
& {[k}
\end{aligned}
$$




$$
\begin{aligned}
= & F \varepsilon_{i}(k)+G C e_{i}(k)-c_{1}\left(1+d_{i}+g_{i}\right)^{-1} T B K Q_{1} C \\
& \times\left[\sum_{j \in \mathcal{N}_{i}} w_{i j}\left(e_{i}(k)-e_{j}(k)\right)+g_{i} e_{i}(k)\right] \\
& -c_{1}\left(1+d_{i}+g_{i}\right)^{-1} T B K Q_{2} \\
& \times\left[\sum_{j \in \mathcal{N}_{i}} w_{i j}\left(\varepsilon_{i}(k)-\varepsilon_{j}(k)\right)+g_{i} \varepsilon_{i}(k)\right] .
\end{aligned}
$$

Let $e=\left(e_{1}^{T}, e_{2}^{T}, \ldots, e_{n}^{T}\right)^{T}$ and $\varepsilon=\left(\varepsilon_{1}^{T}, \varepsilon_{2}^{T}, \ldots, \varepsilon_{n}^{T}\right)^{T}$. From (34), the closed-loop error dynamics can be represented as

$$
\begin{aligned}
& {\left[\begin{array}{l}
e(k+1) \\
\varepsilon(k+1)
\end{array}\right]} \\
& =\left[\begin{array}{cc}
I_{n} \otimes A-c_{1} \Gamma \otimes B K Q_{1} C & -c_{1} \Gamma \otimes B K Q_{2} \\
I_{N} \otimes G C-c_{1} \Gamma \otimes T B K Q_{1} C & I_{n} \otimes F-c_{1} \Gamma \otimes T B K Q_{2}
\end{array}\right] \\
& \times\left[\begin{array}{l}
e(k) \\
\varepsilon(k)
\end{array}\right] \triangleq H\left[\begin{array}{l}
e(k) \\
\varepsilon(k)
\end{array}\right] \text {. }
\end{aligned}
$$

It is easy to see that the leader-following multiagent system achieves consensus if the closed-loop error dynamics system (35) is Schur-stable.

Let $\bar{T}=\left[\begin{array}{cc}I_{n} \otimes I_{m} & 0 \\ -I_{n} \otimes T & I_{n} \otimes I_{m-q}\end{array}\right]$, which is nonsingular, and $\bar{T}^{-1}=$ $\left[\begin{array}{cc}I_{n} \otimes I_{m} & 0 \\ I_{n} \otimes T & I_{n} \otimes I_{m-q}\end{array}\right]$. By step (2) of Algorithm 13, we have

$$
\bar{H} \triangleq \bar{T} H \bar{T}^{-1}=\left[\begin{array}{cc}
I_{n} \otimes A-c_{1} \Gamma \otimes(B K) & -c_{1} \Gamma \otimes\left(B K Q_{2}\right) \\
0 & I_{n} \otimes F
\end{array}\right] .
$$

The matrix $\bar{H}$ is block upper triangular matrix with diagonal block matrix entries $I_{n} \otimes A-c_{1} \Gamma \otimes(B K)$ and $F$. Because $F$ is Schur-stable, the matrix $H$ is Schur-stable if and only if $A-$ $c_{1} \Gamma \otimes(B K)$ is Schur-stable. The rest of the proof is omitted, because it is very similar to the proof of Theorem 7 .

\section{Simulation Example}

In this section, we give an example to illustrate the effectiveness of the obtained result. The multiagent system consists of four agents and one leader, that is, $n=4$. The following agents and leader are, respectively, modeled by the linear dynamics (1) and (2) with system matrices

$$
\begin{gathered}
A=\left[\begin{array}{ccc}
0 & 3 & 0 \\
0 & 0 & 1 \\
-0.2 & 0.2 & 1.1
\end{array}\right], \quad B=\left[\begin{array}{l}
0 \\
0 \\
4
\end{array}\right], \\
C=[0,1,1] .
\end{gathered}
$$

The matrices $L$ and $G$ of the interaction graph $\widehat{\mathscr{G}}$ are given by

$$
L=\left[\begin{array}{cccc}
2 & -1 & -1 & 0 \\
-1 & 2 & -1 & 0 \\
-1 & -1 & 3 & -1 \\
-1 & 0 & -1 & 2
\end{array}\right], \quad G_{d}=\left[\begin{array}{llll}
0 & 0 & 0 & 0 \\
0 & 0 & 0 & 0 \\
0 & 0 & 1 & 0 \\
0 & 0 & 0 & 1
\end{array}\right]
$$

By some simple computations, it is proper to take $c_{0}=0.5768$, $r_{0}=0.5001$. Therefore, take $c_{1}=1.7337$. By solving MDRAE (3) with $\delta=0.2482$, the unique positive definite solution is

$$
P=\left[\begin{array}{ccc}
2.7685 & 2.0965 & -8.6525 \\
2.0965 & 17.0036 & -7.1766 \\
-8.6525 & -7.1766 & 59.3567
\end{array}\right]
$$

Then, the gain matrix can be chosen as

$$
K=\left(I+B^{T} P B\right)^{-1} B^{T} P A=[-0.0499,-0.0593,0.2445] .
$$

The multiagent system adopts the consensus protocols (16) and (17) with randomly initial state. The matrices $F, G$, and $T$ are designed as follows:

$$
\begin{gathered}
F=\left[\begin{array}{ccc}
-0.1 & 0 & 0 \\
1 & -0.2 & 0 \\
0 & 0 & -0.3
\end{array}\right], \quad G=\left[\begin{array}{l}
1 \\
0 \\
3
\end{array}\right], \\
T=\left[\begin{array}{ccc}
0 & 1 & -0.9 \\
0.4041 & 0.3342 & 0.4041 \\
0.6306 & 4.3243 & -0.9459
\end{array}\right] .
\end{gathered}
$$

The state tracking errors showed in Figure 1, which show all following agents can track the leader. As for the reducedorder observer case, the matrices $F, G, T, Q_{1}$, and $Q_{2}$ used in the protocols (27) and (28) can be constructed by Algorithm 13 as follows:

$$
\begin{gathered}
F=\left[\begin{array}{cc}
-0.1 & 0 \\
-0.1 & -0.1
\end{array}\right], \quad G=[4,7]^{T}, \\
T=\left[\begin{array}{ccc}
1.1842 & 3.2895 & 0.5921 \\
0.8535 & 5.6999 & 0.1009
\end{array}\right], \\
Q_{1}=[-0.7031,0.0892,0.9168]^{T}, \\
Q_{2}=\left[\begin{array}{cc}
1.2936 & -0.6232 \\
-0.1972 & 0.2736 \\
0.1972 & -0.2736
\end{array}\right] .
\end{gathered}
$$

With consensus protocols (27) and (28), the state tracking errors showed in Figure 2, which also show all following agents, can track the leader.

\section{Conclusions}

This paper solves a leader-following consensus problem of discrete-time multiagent system with distributed controllers and observers. We provide a general framework for designing 


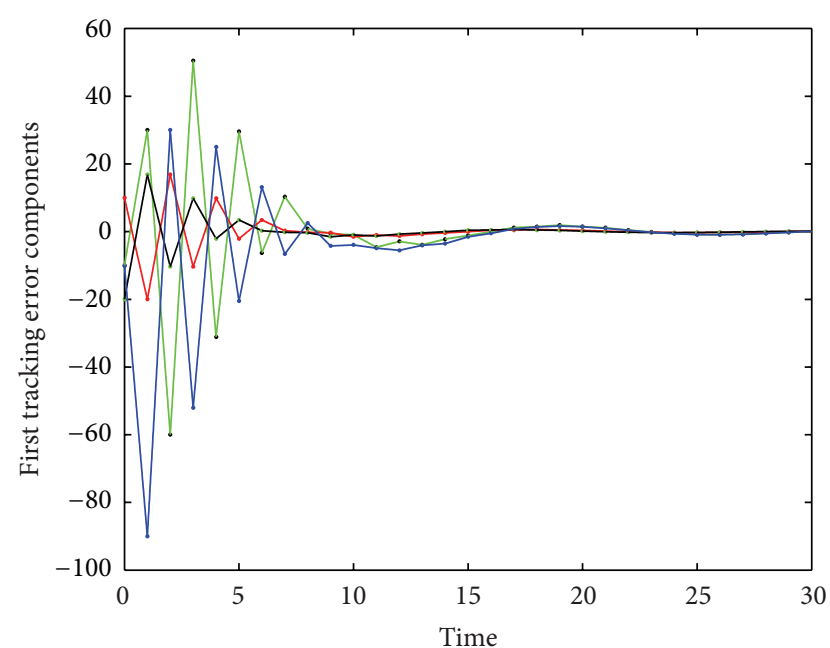

(a)

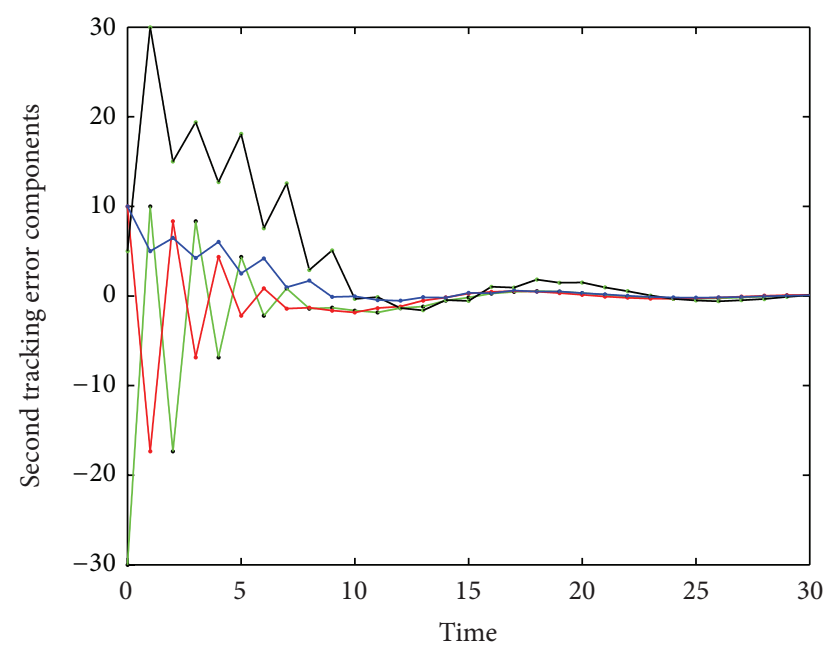

(b)

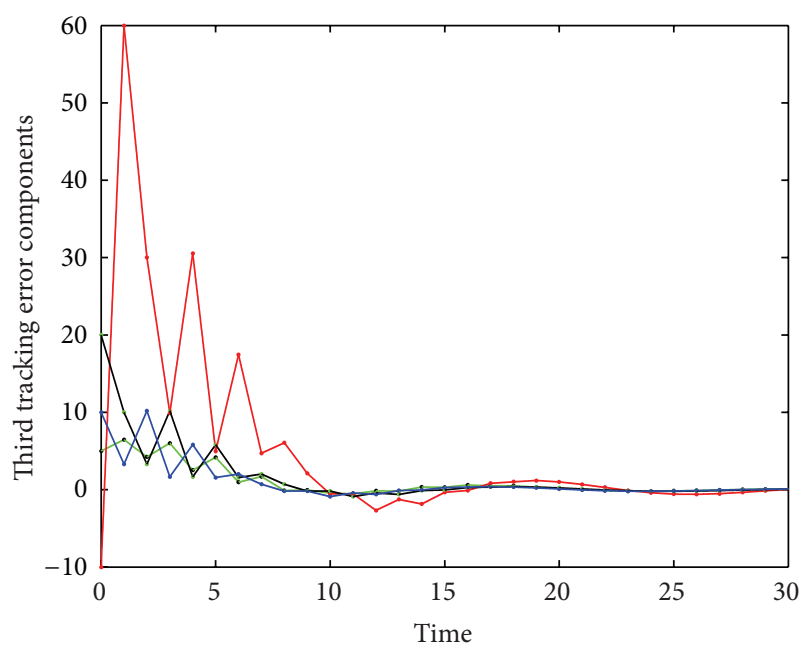

(c)

Figure 1: Error trajectories of three state components with fullorder observer.

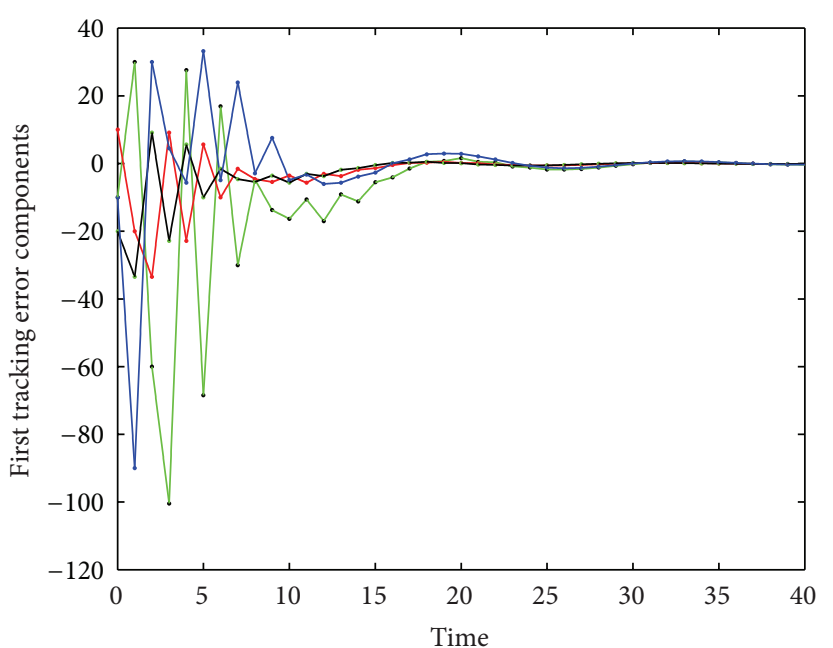

(a)

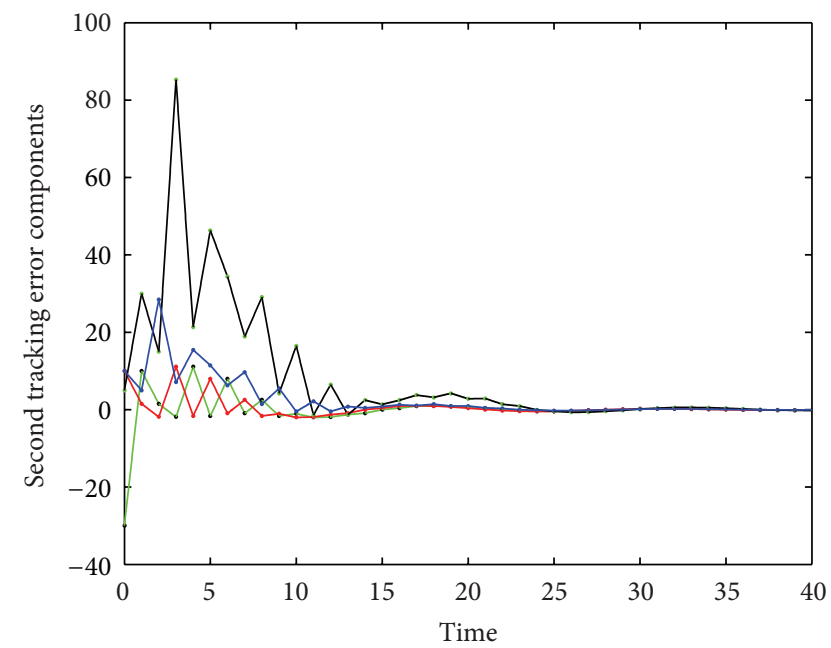

(b)

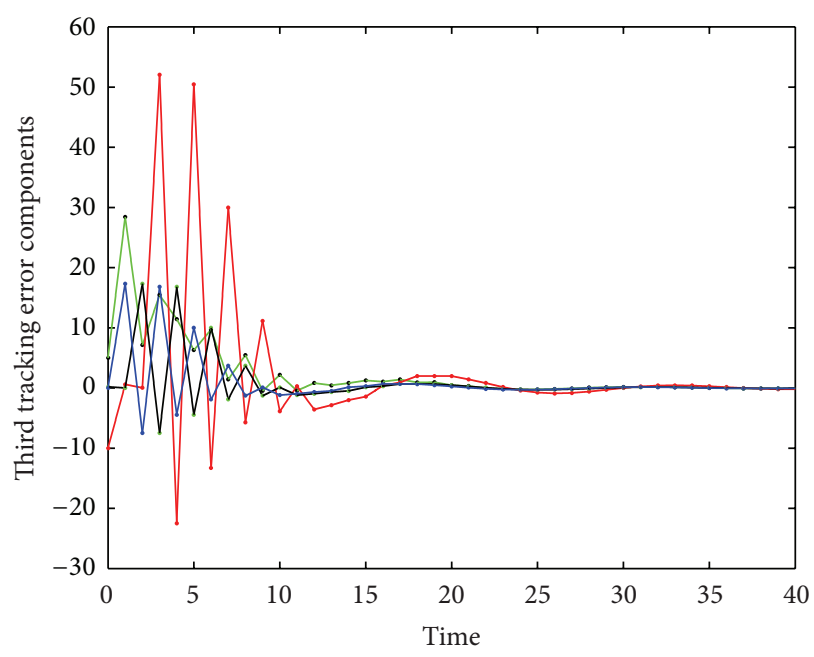

(c)

FIGURE 2: Error trajectories of three state components with reducedorder observer. 
distributed consensus protocols by applying full state feedback information and measured output feedback information. Furthermore, we propose a reduced-order observerbased protocol to solve the leader-following consensus problem. The interconnection topology is modeled by graph, whose connectivity is a key factor to guarantee that the multiagent achieves consensus. The consensus problem is transformed into the stability problem of error dynamical system, which also preserves the property of the separation principle. The gain matrices can be designed by solving the MDARE and the Sylvester equation. Presented results could be generalized to switching and jumping interaction topology in future work.

\section{Acknowledgments}

This work was supported by the Zhejiang Provincial Natural Science Function of China under Grant no. LY13F030048 and the National Natural Science Function of China under Grant nos. $61074123,61174063$.

\section{References}

[1] J. Krause, G. D. Ruxton, and S. Krause, "Swarm intelligence in animals and humans," Trends in Ecology \& Evolution, vol. 25, no. 1, pp. 28-34, 2010.

[2] T. Vicsek, A. Czirk, E. Ben-Jacob, I. Cohen, and O. Shochet, "Novel type of phase transition in a system of self-driven particles," Physical Review Letters, vol. 75, no. 6, pp. 1226-1229, 1995.

[3] S. Kalantar and U. R. Zimmer, "Distributed shape control of homogeneous swarms of autonomous underwater vehicles," Autonomous Robots, vol. 22, no. 1, pp. 37-53, 2007.

[4] S. Yoon and C. Qiao, "Cooperative search and survey using Autonomous Underwater Vehicles (AUVs)," IEEE Transactions on Parallel and Distributed Systems, vol. 22, no. 3, pp. 364-379, 2011.

[5] Z. Huang, Q. Song, and C. Feng, "Multistability in networks with self-excitation and high-order synaptic connectivity," IEEE Transactions on Circuits and Systems I, vol. 57, no. 8, pp. 21442155, 2010.

[6] E. Kaslik and S. Sivasundaram, "Multiple periodic solutions in impulsive hybrid neural networks with delays," Applied Mathematics and Computation, vol. 217, no. 10, pp. 4890-4899, 2011.

[7] W. Yu, G. Chen, Z. Wang, and W. Yang, "Distributed consensus filtering in sensor networks," IEEE Transactions on Systems, Man, and Cybernetics B, vol. 39, no. 6, pp. 1568-1577, 2009.

[8] A. Jadbabaie, J. Lin, and A. S. Morse, "Coordination of groups of mobile autonomous agents using nearest neighbor rules," IEEE Transactions on Automatic Control, vol. 48, no. 6, pp. 988-1001, 2003.

[9] W. Ren and R. W. Beard, "Consensus seeking in multiagent systems under dynamically changing interaction topologies," IEEE Transactions on Automatic Control, vol. 50, no. 5, pp. 655661, 2005.

[10] R. Olfati-Saber and R. M. Murray, "Consensus problems in networks of agents with switching topology and time-delays," IEEE Transactions on Automatic Control, vol. 49, no. 9, pp. 15201533, 2004.
[11] R. Olfati-Saber, J. A. Fax, and R. M. Murray, "Consensus and cooperation in networked multi-agent systems," Proceedings of the IEEE, vol. 95, no. 1, pp. 215-233, 2007.

[12] Y. Hong, J. Hu, and L. Gao, "Tracking control for multiagent consensus with an active leader and variable topology," Automatica, vol. 42, no. 7, pp. 1177-1182, 2006.

[13] J. Hu and Y. Hong, "Leader-following coordination of multiagent systems with coupling time delays," Physica A, vol. 374, no. 2, pp. 853-863, 2007.

[14] Y. Hong, G. Chen, and L. Bushnell, "Distributed observers design for leader-following control of multi-agent networks," Automatica, vol. 44, no. 3, pp. 846-850, 2008.

[15] W. Ni and D. Cheng, "Leader-following consensus of multiagent systems under fixed and switching topologies," Systems \& Control Letters, vol. 59, no. 3-4, pp. 209-217, 2010.

[16] Z.-J. Tang, T.-Z. Huang, J.-L. Shao, and J.-P. Hu, "Leaderfollowing consensus for multi-agent systems via sampled-data control," IET Control Theory \& Applications, vol. 5, no. 14, pp. 1658-1665, 2011.

[17] L. Gao, X. Zhu, and W. Chen, "Leader-following consensus problem with an accelerated motion leader," International Journal of Control, Automation, and Systems, vol. 10, no. 5, pp. 931-939, 2012.

[18] Y. Hong and X. Wang, "Multi-agent tracking of a highdimensional active leader with switching topology," Journal of Systems Science and Complexity, vol. 22, no. 4, pp. 722-731, 2009.

[19] L. Gao, Y. Tang, W. Chen, and H. Zhang, "Consensus seeking in multi-agent systems with an active leader and communication delays," Kybernetika, vol. 47, no. 5, pp. 773-789, 2011.

[20] Z. Li, Z. Duan, G. Chen, and L. Huang, "Consensus of multiagent systems and synchronization of complex networks: a unified viewpoint," IEEE Transactions on Circuits and Systems I, vol. 57, no. 1, pp. 213-224, 2010.

[21] Z. Li, Z. Duan, and G. Chen, "Dynamic consensus of linear multi-agent systems," IET Control Theory and Applications, vol. 5, no. 1, pp. 19-28, 2011.

[22] H. Zhang, F. L. Lewis, and A. Das, "Optimal design for synchronization of cooperative systems: state feedback, observer and output feedback," IEEE Transactions on Automatic Control, vol. 56, no. 8, pp. 1948-1952, 2011.

[23] K. Hengster-Movric and F. Lewis, "Cooperative observers and regulators for discrete-time multiagent systems," International Journal of Robust and Nonlinear Control, vol. 23, no. 14, pp. 1545-1562, 2013.

[24] L. Gao, X. Zhu, W. Chen, and H. Zhang, "Leader-following consensus of linear multiagent systems with state observer under switching topologies," Mathematical Problems in Engineering, vol. 2013, Article ID 873140, 12 pages, 2013.

[25] H. Li, "Observer-type consensus protocol for a class of fractional-order uncertain multiagent systems," Abstract and Applied Analysis, vol. 2012, Article ID 672346, 18 pages, 2012.

[26] Z. Li, X. Liu, P. Lin, and W. Ren, "Consensus of linear multiagent systems with reduced-order observer-based protocols," Systems \& Control Letters, vol. 60, no. 7, pp. 510-516, 2011.

[27] Y. Zhang, L. Gao, and C. Tong, "On distributed reduced-order observer-based protocol for linear multiagent consensus under switching topology," Abstract and Applied Analysis, vol. 2013, Article ID 793276, 13 pages, 2013.

[28] J. H. Seo, H. Shim, and J. Back, "Consensus of high-order linear systems using dynamic output feedback compensator: low gain approach," Automatica, vol. 45, no. 11, pp. 2659-2664, 2009. 
[29] Y. Liu and Y. Jia, " $H_{\infty}$ consensus control of multi-agent systems with switching topology: a dynamic output feedback protocol," International Journal of Control, vol. 83, no. 3, pp. 527-537, 2010.

[30] L. Gao, C. Tong, and L. Wang, " $H^{\infty}$ dynamic output feedback consensus control for discrete-time multi-agent systems with switching topology," The Arabian Journal for Science and Engineering, 2013.

[31] Z. Huang, X. Wang, and C. Feng, "Multiperiodicity of periodically oscillated discrete-time neural networks with transient excitatory self-connections and sigmoidal nonlinearities," IEEE Transactions on Neural Networks, vol. 21, no. 10, pp. 1643-1655, 2010.

[32] Z. Huang, X. Wang, and M. Sannay, "Self-excitation of neurons leads to multiperiodicity of discrete-time neural networks with distributed delays," Science China, vol. 54, no. 2, pp. 305-317, 2011.

[33] E. Kaslik and S. Sivasundaram, "Impulsive hybrid discretetime Hopfield neural networks with delays and multistability analysis," Neural Networks, vol. 24, no. 4, pp. 370-377, 2011.

[34] Y. Cao and W. Ren, "Sampled-data discrete-time coordination algorithms for double-integrator dynamics under dynamic directed interaction," International Journal of Control, vol. 83, no. 3, pp. 506-515, 2010.

[35] K. You and L. Xie, "Network topology and communication data rate for consensusability of discrete-time multi-agent systems," IEEE Transactions on Automatic Control, vol. 56, no. 10, pp. 2262-2275, 2011.

[36] F. Xiao and L. Wang, "Dynamic behavior of discrete-time multiagent systems with general communication structures," Physica A, vol. 370, no. 2, pp. 364-380, 2006.

[37] F. Xiao and L. Wang, "Consensus protocols for discrete-time multi-agent systems with time-varying delays," Automatica, vol. 44, no. 10, pp. 2577-2582, 2008.

[38] P. Lin and Y. Jia, "Consensus of second-order discrete-time multi-agent systems with nonuniform time-delays and dynamically changing topologies," Automatica, vol. 45, no. 9, pp. 21542158, 2009.

[39] J. Qin and H. Gao, "A sufficient condition for convergence of sampled-data consensus for double-integrator dynamics with nonuniform and time-varying communication delays," IEEE Transactions on Automatic Control, vol. 57, no. 9, pp. 2417-2422, 2012.

[40] Y. Su and J. Huang, "Two consensus problems for discretetime multi-agent systems with switching network topology," Automatica, vol. 48, no. 9, pp. 1988-1997, 2012.

[41] K. Hengster-Movric, K. You, F. L. Lewis, and L. Xie, "Synchronization of discrete-time multi-agent systems on graphs using Riccati design," Automatica, vol. 49, no. 2, pp. 414-423, 2013.

[42] K. You and L. Xie, "Coordination of discrete-time multi-agent systems via relative output feedback," International Journal of Robust and Nonlinear Control, vol. 21, no. 13, pp. 1587-1605, 2011.

[43] X. Xu, S. Chen, W. Huang, and L. Gao, "Leader-following consensus of discrete-time multi-agent systems with observerbased protocols," Neurocomputing, vol. 118, no. 22, pp. 334-341, 2013.

[44] L. Scardovi and R. Sepulchre, "Synchronization in networks of identical linear systems," Automatica, vol. 45, no. 11, pp. 25572562, 2009.

[45] B. Sinopoli, L. Schenato, M. Franceschetti, K. Poolla, M. I. Jordan, and S. S. Sastry, "Kalman filtering with intermittent observations," IEEE Transactions on Automatic Control, vol. 49, no. 9, pp. 1453-1464, 2004.
[46] L. Schenato, B. Sinopoli, M. Franceschetti, K. Poolla, and S. S. Sastry, "Foundations of control and estimation over lossy networks," Proceedings of the IEEE, vol. 95, no. 1, pp. 163-187, 2007.

[47] R. A. Horn and C. R. Johnson, Matrix Analysis, Cambridge University Press, New York, NY, USA, 1985. 


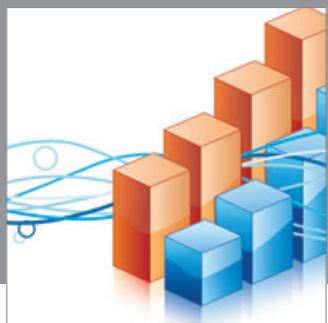

Advances in

Operations Research

mansans

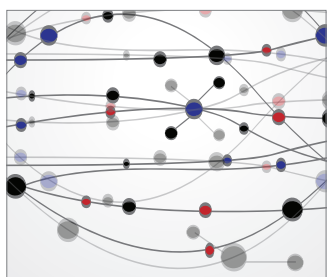

The Scientific World Journal
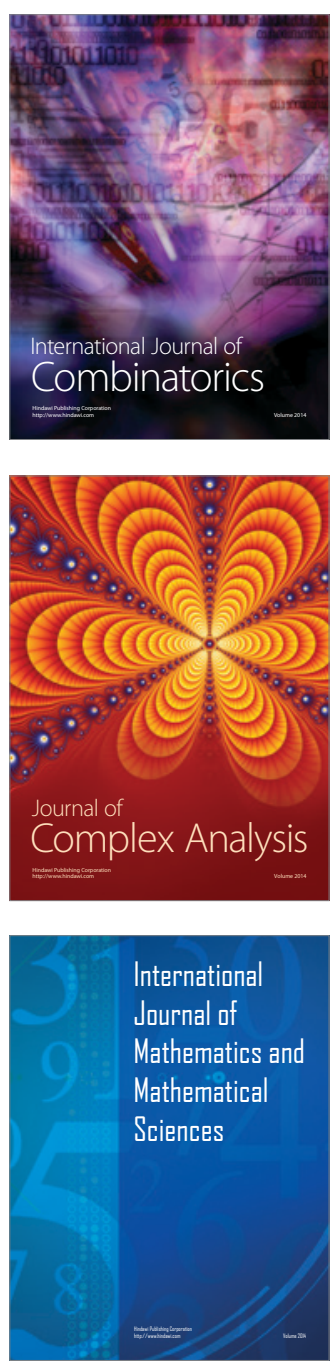
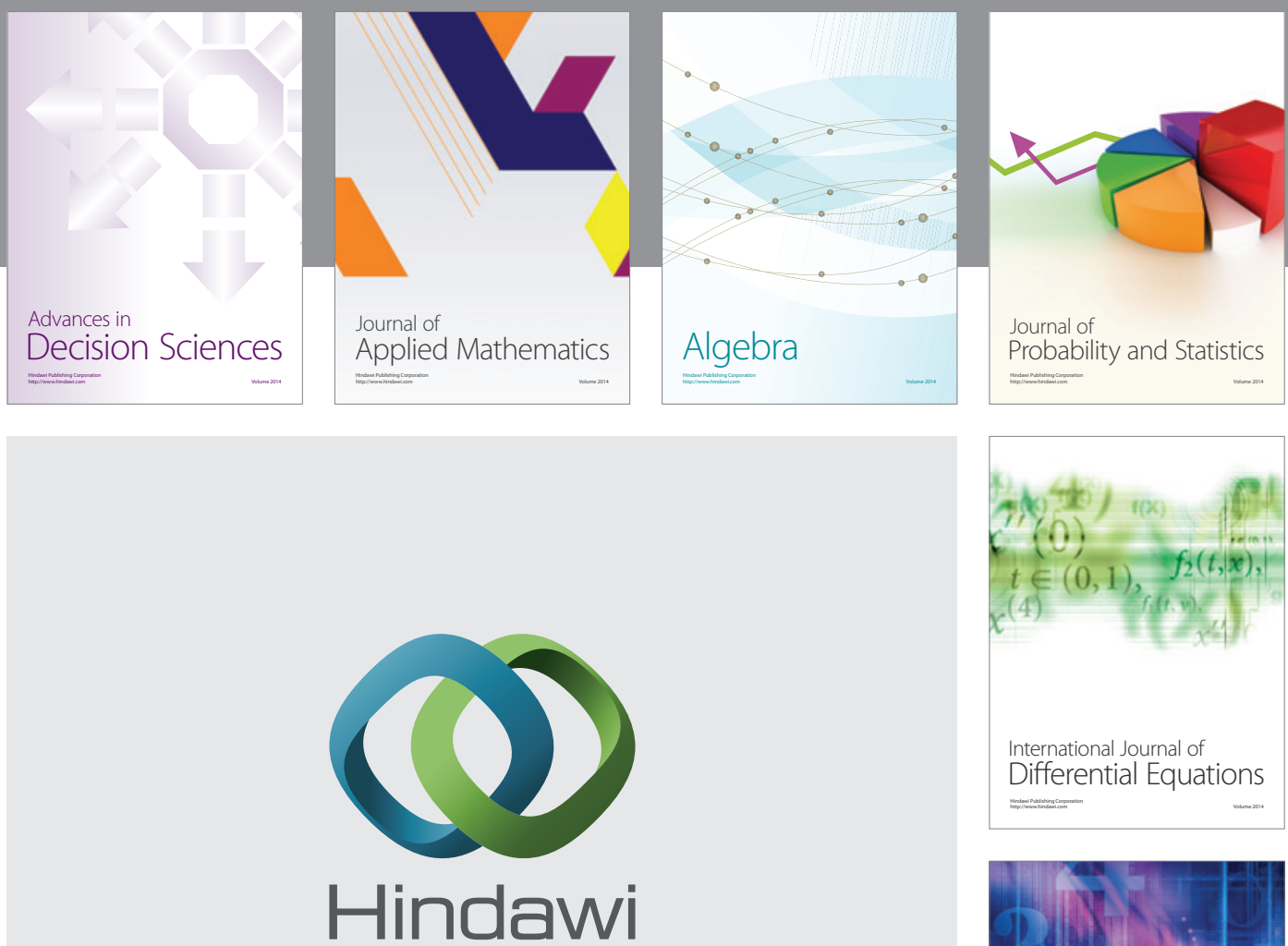

Submit your manuscripts at http://www.hindawi.com
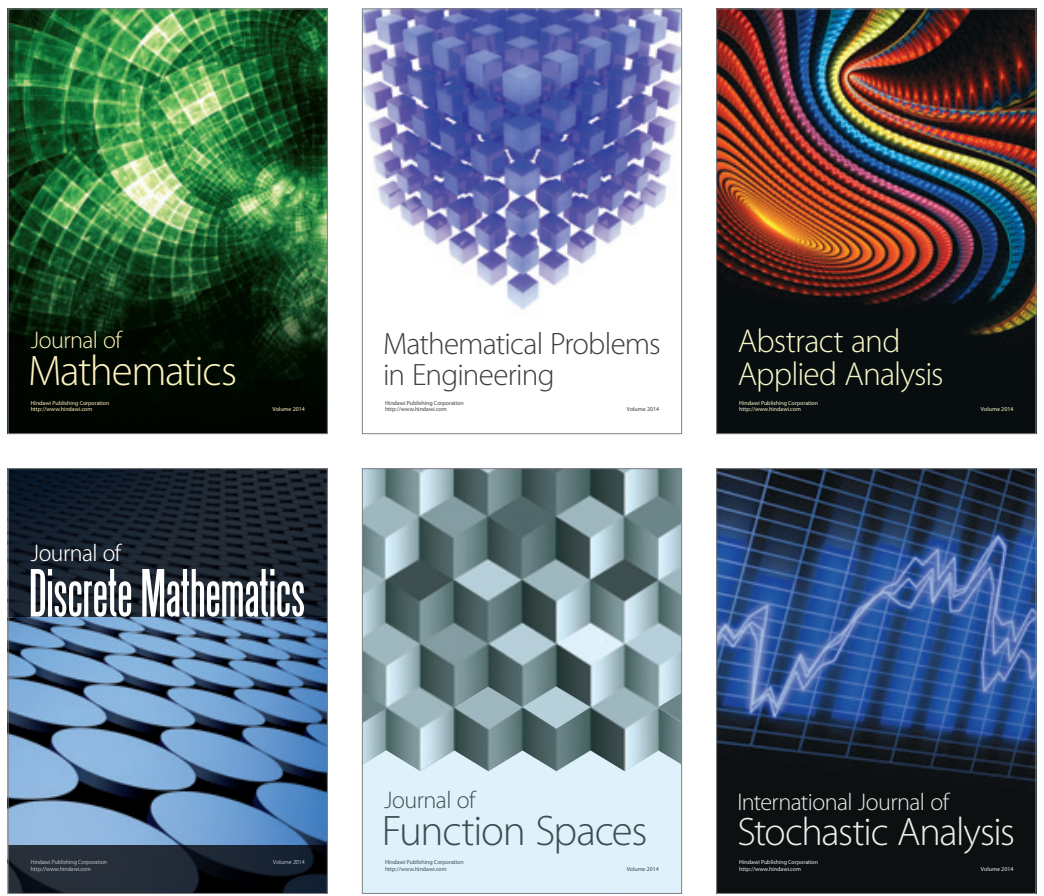

Journal of

Function Spaces

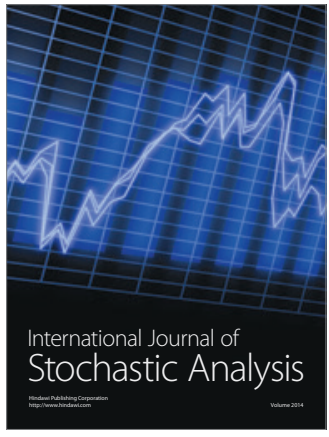

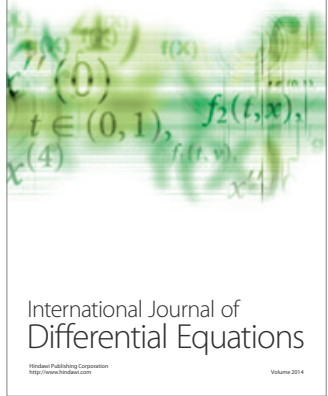
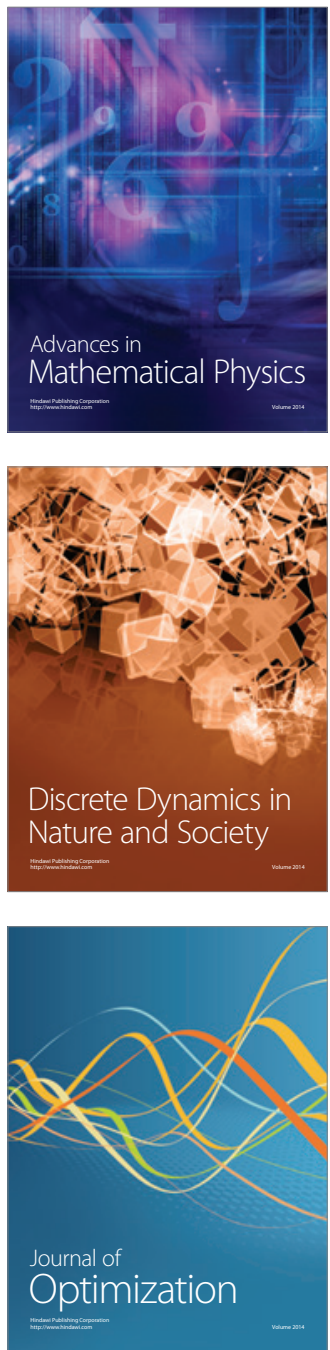